Innovations in Incidence Geometry

Volume 15 (2017), Pages 265-286

ISSN 1781-6475

\title{
Parapolar spaces with the "Haircut" Axiom
}

\author{
Ernest E. Shult \\ To a great friend and mathematician, J. A. Thas
}

\begin{abstract}
In this paper, a generalization of a well-known result of Cohen and Cooperstein [4] on strong parapolar spaces of symplectic rank at least three, with only finite-dimensional singular subspaces, is presented. In contrast with the aforementioned theorem, we do not assume that symplecta posses a uniform symplectic rank, we drop the assumption that the considered spaces are strong parapolar spaces, and we replace axiom (CC) by the much more general "haircut axiom."
\end{abstract}

Note added in proof. During the refereeing process of the present paper, Ernie Shult's health deteriorated up to a point that he was no longer sure that he would be able to do the final editing. He passed the ${ }_{\mathrm{H}} \mathrm{T} \mathrm{X}$-files on to me, and asked me to do it. I tried to do it the best I could. He never saw the final version.

- Koen Thas

Keywords : building, exceptional geometry, Grassmann space, haircut axiom, parapolar space

MSC 2010: 51A50, 51B25, 51E24, 51M35

\section{Introduction}

The important role of symplecta in characterizing certain exceptional geometries emerged in early papers of B. Cooperstein [5]. This paradigm was opened further by two fundamental theorems which revealed the role of a broad class of geometries with symplecta — called parapolar spaces—as a stage upon which the geometries derived from buildings might be characterized by simple axioms on points and lines.

In [2], Arjeh Cohen characterized Grassmann spaces this way: 
Theorem 1.1 (Cohen). Let $\Gamma=(\mathcal{P}, \mathcal{L})$ be a strong parapolar space of constant symplectic rank 3 , with all singular subspaces (necessarily projective spaces) having finite rank. Assume $\Gamma$ possesses the following property:

(H3) If $x$ is a point and $S$ is a symplecton not containing $x$, then $x^{\perp} \cap S$, the set of points of $S$ which are collinear with $x$, is either the empty set, a single point, or is a projective plane. In other words, it is never a single line.

Then one of two possibilities exist:

(1) $\Gamma$ is a Grassmann space $A_{n, k}, 1<k \leq(n+1) / 2$.

(2) $\Gamma$ is $A_{2 n-1, n} /\langle\sigma\rangle$, a homomorphic image of a Grassmann space, whose elements are orbits of a polarity $\sigma$ of Witt index at most $n-5$.

A full description of the terminology used in this and subsequent theorems is given in Section 2.

A key ingenious argument in Cohen's paper produced a proof that all the symplecta of $\Gamma$ were oriflamme-that is, any line of a symplecton lies in exactly two planes of the symplecton. The argument produced a peculiar sort of generalized quadrangle which must exist as a substructure of the point-residual of any symplecton. The quadrangle in question contains a spread of lines $\mathcal{S}$ such that any two lines of the spread generate a grid whose lines parallel to the generating lines belong to $\mathcal{S}$. Moreover, if $\mathcal{G}$ denotes the collection of such grids, then the quadrangle is either itself a grid or else the incidence structure $(\mathcal{S}, \mathcal{G})$ is a projective plane. The statement that the latter is impossible has come to be known as "Cohen's Lemma." Cohen's original proof of it invoked Tits's classification of all polar spaces of polar rank three. A somewhat simpler proof invoked only the property that rank three polar spaces were Moufang [11]. Recently, J. Schillewaert and H. Van Maldeghem [8] showed the non-existence of such a quadrangle without invoking any further hypotheses whatsoever.

The second theorem ${ }^{1}$ opening up the parapolar paradigm was the following:

Theorem 1.2 (Cohen-Cooperstein [4]). Let $\Gamma$ be a strong parapolar space of symplectic rank at least three with the various projective ranks of all singular subspaces finite. Assume

(i) All symplecta have the same polar rank $r$.

(ii) $\Gamma$ possesses the following restriction on point-symplecton pairs:

\footnotetext{
${ }^{1}$ For the historical record, the above theorem was also accompanied by another theorem that involved parapolar spaces of constant symplectic rank which were not strong, with assumption (ii) replaced by the assumption that $x^{\perp} \cap S$ is equal to the empty set, a line or a maximal singular subspace of $S$. Although that theorem inspired a series of papers on long-root geometries, $[6,10]$ it happens to be outside of the realm of this paper.
} 
(CC) If $x$ is a point not incident with a symplecton $S$, then $x^{\perp} \cap S$ is either the empty set, a single point, or a maximal singular subspace of $S$ (necessarily a $\mathrm{PG}(r-1)$ ).

Then one of the following holds:

(1) $r=3$ and $\Gamma$ is either $A_{n, k}$ or $A_{2 n-1, n} /\langle\sigma\rangle$ as in Theorem 1.1.

(2) $r=4$ and $\Gamma$ is a half-spin geometry $D_{n, n}$.

(3) $r=5$ and $\Gamma$ is the exceptional geometry $E_{6.1}$.

(4) $r=6$ and $\Gamma$ is the exceptional geometry $E_{7,7}$.

Note. The numbering of the nodes of the exceptional Dynkin diagrams follows that of Bourbaki: the end node of the middle-sized arm is numbered "1," the end-node of the shortest arm is " 2 ," and the numbering proceeds along the path of the remaining nodes with " 3 " next to " 1 " and ending at the end of the long arm with label " $n$." (In the spherical case, $n=6,7,8$.)

The main theorem of this paper generalizes Theorem 1.2 in three ways:

1. It drops the assumption that symplecta possess a uniform symplectic rank.

2. It drops the assumption that $\Gamma$ is a strong parapolar space.

3. Axiom $(\mathrm{CC})$ is replaced by the much more general "haircut axiom" $(\mathrm{H})$ described below.

On the other hand, one must pay for this generality by allowing some rather bizarre geometries to intrude into the list of possible conclusion geometries. Some of these are "extreme" parapolar spaces (see Subsection 2.3), some are homomorphic images of possibly non-spherical buildings of type $E_{n, 1}$ and others, such as $E_{8,1}$ and $E_{8,2}$, although derived from a spherical building, do not appear in any previous point-line characterization theorem, and do not seem to be fully analyzed at the present time.

Theorem 1.3. (Main Theorem) Suppose $\Sigma$ is a parapolar space of sympletic rank at least three. Although we have not assumed constant symplectic rank, we shall assume the following:

(i) Each singular space possesses a finite projective dimension. Moreover, there exists an upper bound to the polar rank of a symplecton, and all symplecta have polar rank at least three.

(ii) We assume the "hair-cut axiom":

(H) If $S$ is a symplecton and $x$ is a point not in $S$, then $x^{\perp} \cap S$ cannot be a hyperplane of a maximal singular subspace of $S$.

(iii) $\Sigma$ is a locally connected space. 
Then $\Sigma$ possesses a uniform symplectic rank $k$ and one of the following occurs:

(1) $k=3$ and $\Sigma$ is either a Grassmann space $A_{n, k}$, or a homomorphic image $A_{2 n-1, n} /\langle\sigma\rangle$, where $\sigma$ is a polarity of $A_{2 n-1}$ of Witt index at most $n-5$.

(2) $k=4$ and $\Sigma$ is a $Y_{1}$-geometry or a twisted version thereof (see Subsection 3.2). In the latter case $\Sigma$ is extreme. If the parapolar space $\Sigma$ is neither extreme or strong, then $\Sigma$ is the long-root geometry $E_{6,2}$. If $\Sigma$ is a strong parapolar space, then $\Sigma$ is a half-spin geometry of type $D_{n, n}, n \geq 5$.

(3) $k=5$ and $\Sigma$ belongs to a $Y_{1,2, m}$-diagram and is a homomorphic image of the building geometry $E_{m+4,1}$. If $m=4$ then $\Sigma$ is the building geometry $E_{8,1}{ }^{2}$ If $m=3$ then $\Sigma$ is the long-root geometry $E_{7,1}$. If $\Sigma$ is a strong parapolar space, then $m=2$ and $\Sigma$ is the Lie incidence geometry $E_{6,1}$.

(4) $k=6$ and $\Sigma$ is the geometry $E_{7,7}$, a strong parapolar space.

(5) $k=7$ and $\Sigma$ is the long-root geometry $E_{8,8}$.

Conversely, all of the listed geometries satisfy the hypotheses.

\section{Basic terminology}

\subsection{Point-line geometries}

A point-line geometry is a set of points $\mathcal{P}$ together with a collection $\mathcal{L}$ of subsets of $\mathcal{P}$ of size at least three called lines. Two distinct points $x$ and $y$ are said to be collinear if they are both contained in a common line. In this way each pointline geometry $(\mathcal{P}, \mathcal{L})$ is associated with a point-collinearity graph $\Delta=(\mathcal{P}, \sim)$ whose vertices are the points, two being adjacent if and only if they are collinear. In this graph, for each point $x$, the symbol $x^{\perp}$ denotes the set consisting of point $x$ together with all points which are collinear with it. We also write $X^{\perp}$ for the set $\cap_{x \in X} x^{\perp}$, where $X \subseteq \mathcal{P}$. The point-line geometry $\Gamma=(\mathcal{P}, \mathcal{L})$ is said to be connected if and only if the graph $\Delta$ is connected. The length of a shortest path in $\Delta$ connecting two points is denoted $d(x, y)$, so in this way $\Delta$ provides a distance metric $d: \mathcal{P} \times \mathcal{P} \rightarrow \mathbb{N}$. The diameter of any graph is the maximal distance (if it exists) between any two of its vertices. A point-line geometry $(\mathcal{P}, \mathcal{L})$ is said to have point-diameter $m$ if and only if its associated point-collinearity graph has this diameter.

A subspace of a point-line geometry $(\mathcal{P}, \mathcal{L})$ is a subset $A$ of points with the

\footnotetext{
${ }^{2}$ In the table of parapolar space characterizations on page 722 of the survey article [3], a characterization in [4] uses the notation $E_{8,1}$ instead of $E_{8,8}$. It is correctly listed as $E_{8,8}$ in sec. 6.11, p. 720 , of the same article. As far as is known to the author, $E_{8,1}$ has not heretofore been characterized as a parapolar space.
} 
property that if $L \in \mathcal{L}$ and $L \cap A$ contains at least two points, then $L \subseteq A$. Thus the points of any subspace together with the lines contained in it, can itself be regarded as a point-line geometry and is thus subject to any of the adjectives we attach to point-line geometries. The intersection of subspaces is again a subspace, and the smallest subspace containing a set $X$ is denoted $\langle X\rangle$. A subspace $A$ is said to be convex if and only if, for any shortest possible path connecting any two of its points, all intermediate points of such a path also lie in $A$. A subspace is said to be a singular subspace if any two of its points are collinear.

A point-line geometry $\Gamma=(\mathcal{P}, \mathcal{L})$ is said to be a gamma space if and only if, for each of its points $p$, the set $p^{\perp}$ is a subspace of $\Gamma$; $\Gamma$ is said to be a partial linear space if and only if any two distinct lines intersect in at most one point. A singular space which is also a partial linear space is simply called a linear space.

\subsection{Projective spaces and polar spaces}

We describe here two very important types of point-line geometries, projective spaces and polar spaces. A projective plane is a linear space in which any two lines intersect at a point. A projective space is a linear space in which any two lines that intersect at a point lie in a subspace that is a projective plane. The famous Veblen-Young Theorem ([13]) asserts that any projective space that properly contains a projective plane is in fact isomorphic to the geometry $\mathrm{PG}(V)$ whose points and lines are the 1- and 2-dimensional vector subspaces of a vector space $V$ over some division ring. This allows us to attach a rank (or projective dimension) to each projective space. Thus a single point has rank 0 , a single line has rank 1 , a projective plane has rank 2 , and the rank of $\mathrm{PG}(V)$ is $\operatorname{dim} V-1$, where $\operatorname{dim} V$ denotes the vector space dimension of $V$.

For the purposes of this paper ${ }^{3}$ a polar space is a point-line geometry $(\mathcal{P}, \mathcal{L})$ with these two properties:

(i) $\mathcal{L}$ is non-empty and for any point $p$ that does not lie in a line $L$, either $p$ is collinear with exactly one point of $L$, or is collinear with all points of $L$.

(ii) No point is collinear with all remaining points.

It is obvious from the definition that a polar space cannot be a single point, and that it is a connected gamma space of point diameter two. The following however is not so obvious:

Theorem 2.1. A polar space is a partial linear space and all its singular subspaces are projective spaces.

\footnotetext{
${ }^{3}$ In the general literature, what we define is called a non-degenerate polar space.
} 
If all these singular subspaces of a polar space have finite projective dimension, then the maximal singular subspaces all have the same projective dimension $m$. In that case the polar space is said to have polar rank $m+1$. A polar space of polar rank two is called a generalized quadrangle.

\subsection{Parapolar spaces}

In a point-line geometry $\Gamma$, a convex subspace that happens to be a polar space is called a symplecton. If the symplecton has polar rank two-that is, it is a generalized quadrangle- then we refer to it as a quad.

Because of the convexity, if $x$ is a point not in the symplecton $S$, then $x^{\perp} \cap S$ is a clique-and, if $\Gamma$ is a gamma space, it is a singular subspace.

A parapolar space is a connected gamma space $\Gamma=(\mathcal{P}, \mathcal{L})$ with the following properties:

1. Every line of $\mathcal{L}$ lies in a symplecton.

2. $\Gamma$ is not itself a polar space.

3. For any two points $x$ and $y$ at distance two in $\Delta$ one of the following occurs:

(a) $x^{\perp} \cap y^{\perp}$ consists of a single point $z(x, y)$. (In this case $\{x, y\}$ is called a special pair and $z(x, y)$ is called the center of the special pair.)

(b) $\{x, y\}$ is contained in a symplecton. (In this case $\{x, y\}$ is called a polar pair.)

If all symplecta of the parapolar space $\Gamma$ have polar rank $r$ or higher, we say that $\Gamma$ has symplectic rank at least $r$.

Theorem 2.2. If $\Gamma$ is a parapolar space of symplectic rank at least 3, then any singular subspace space generated by a point and a line lies in some symplecton and so is a line or a projective plane. It follows that every singular subspace of $\Gamma$ is a projective space.

Proof. This is Theorem 17.2.3 of [9].

A parapolar subspace is said to be locally connected if, for any two distinct lines $L$ and $N$ incident with a point $p$, their exists a finite sequence of projective planes $\pi_{1}, \ldots, \pi_{s}$ on the point $p$ such that $L \subseteq \pi_{1}, N \subseteq \pi_{s}$, and $\pi_{j} \cap \pi_{j+1}$ is a line on $p$. If this can be done with $s=1$ then $\langle L \cup N\rangle$ is a projective plane. Choose $(x, y) \in L \times N$ so that neither $x$ nor $y$ is equal to $p$. If $L$ and $N$ do not lie in a plane and $s=2$, then the convex closure $\langle\langle L, N\rangle\rangle$ is a symplecton, and $\{x, y\}$ is a polar pair. If we cannot find such a sequence of planes of length $s=2$ then 
$\{x, y\}$ is a special pair. If such a sequence of length $s=3$ cannot be found then the special pair $\{x, y\}$ is called an extreme pair.

A parapolar space without special pairs is called a strong parapolar space. An extreme parapolar space is one that contains extreme pairs. Some of the geometries in the conclusion of the main theorem of this paper are extreme parapolar spaces.

Now assume $\Sigma$ is a parapolar space such that every singular subspace is a projective space and that every line lies in a projective plane. If $p$ is a point of $\Sigma$, the point-residual at $p$ is the geometry of all lines and planes of $\Sigma$ which are incident with the point $p$ and is denoted $\operatorname{Res}_{\Sigma}(p)$. Similarly, if $A$ is a singular subspace of $\Sigma$ which has codimension at least 2 in some other singular spaces, the residue $\Sigma(A)$ is the geometry of singular subspaces in which $A$ has codimension at most two, with containment as incidence. This geometry is merely an incidence system of points and lines; it need not be a parapolar space or even be connected.

There will be frequent appeals to some very elementary facts relating a parapolar space and its point-residuals:

Lemma 2.3. (1) If $S$ is a symplecton of polar rank at least 3 in a parapolar space $\Sigma$, and $p$ is a point of $\Sigma$, then the collection of lines and planes of $S$ that contain $p$ forms a symplecton $\bar{S}$ of the point-residual $\operatorname{Res}_{\Sigma}(p)$.

(2) If $\Sigma$ is a parapolar space of symplectic rank at least 3 then each point-residual $\Sigma(p):=\operatorname{Res}_{\Sigma}(p)$ has the property that each of its pairs of points at distance two lies in a symplecton of $\Sigma(p)$. This symplecton might be a quad. In particular if the point-residual $\Sigma(p)$ is itself a parapolar space, then it is a strong parapolar space.

(3) If $\Sigma$ is a strong parapolar space of symplectic rank at least 3, then each of its point-residuals have point-diameter 2.

We list here three important results on parapolar spaces which we shall use.

Lemma 2.4. Suppose $\Sigma$ is a parapolar space of symplectic rank at least 3. Then the following statements are equivalent:

(i) $\Sigma$ possesses the property $(H)$.

(ii) Every point-residual $\operatorname{Res}_{\Sigma}(p)$ of $\Sigma$ also has the property $(H)$.

Proof. (i) $\Rightarrow$ (ii). Suppose $\Sigma$ has property (H) and select a point $p$. Suppose $S$ is a symplecton containing $p$. Then by part 1 of the preceding Lemma, the lines and planes of $S$ which contain $p$ form a symplecton $S_{p}$ of the point-residual of polar rank at least two. Select a line $L=p x$ of $\Sigma$ which does not lie in $S_{p}$. Now, $x^{\perp} \cap S$ is a singular subspace $U$ of $S$ which 
contains $p$ and its lines and planes on $p$ form a singular subspace $U_{p}$ of the symplecton $S_{p}$. By hypothesis $U$ is not a hyperplane of a maximal singular subspace of $S$, and so $U_{p}$ cannot be a hyperplane of a maximal singular subspace of $S_{p}$.

(ii) $\Rightarrow$ (i). Suppose the property (H) failed for $\Sigma$. Then there exists a pair $(x, S)$ where $x$ is a point not in the symplecton $S$, and $x^{\perp} \cap S$ is a hyperplane $H$ of a maximal singular subspace of $S$. Now choose $p \in H$. Then in $\operatorname{Res}_{\Sigma}(p)$ one sees that $(x p)^{\perp} \cap S_{p}=H_{p}$ is a hyperplane of a maximal singular subspace of $S_{p}$. That is, $(H)$ fails for the residue $\operatorname{Res}_{\Sigma}(p)$. Thus if (i) is false, then statement (ii) is false. The proof is complete.

Lemma 2.5. Suppose $\Sigma$ is a locally connected parapolar space with the following property:

(K) If $x$ is a point and $S$ is a symplecton of polar rank at least 3 , then $x^{\perp} \cap S$ is never just a line.

Then $\Sigma$ is a strong parapolar space of symplectic rank at least 3.

Proof. Suppose $(x, y)$ is a pair of points of $\Sigma$ at distance two and let $p$ be a point in $x^{\perp} \cap y^{\perp}$. Since $\Sigma$ is locally connected, there exists a sequence of projective planes $\left(\pi_{1}, \pi_{2}, \ldots, \pi_{n}\right)$, each containing the point $p$, such that $x p \subset \pi_{1}, p y \subset \pi_{n}$, and $\pi_{i} \cap \pi_{i+1}=L_{i}$, a line, $i=1,2, \ldots, n-1$. We write $L_{0}=x p$ and $L_{n}=p y$. On each $L_{i}$ we select a point $y_{i}$ distinct from $p$, setting $x_{0}=x$ and $x_{n}=y$. Among such sequences we can suppose we have chosen one such that $n$ is as small as possible. Of course $n>1$.

By way of contradiction, we suppose $n>2$. Since $\left(y_{n-2}, y_{n}\right)$ is a polar pair, these points lie in a symplecton $S$. Now, by the minimality of $n, y_{n-3}$ does not lie in the symplecton $S$ of rank at least three, while $y_{n-3}^{\perp} \cap S$ contains the line $L_{n-2}$. By hypothesis (K), $y_{n-3}^{\perp} \cap S$ contains a plane $\pi$. Then there is a line $N$ in $L_{n}^{\perp} \cap \pi$, by the fact that $S$ is a polar space. Now the sequence $\left(\pi_{1}, \ldots, \pi_{n-3},\left\langle L_{n-3}, N\right\rangle,\left\langle N, L_{n}\right\rangle\right)$ is a sequence of planes on $p$ of length $n-1$ connecting $L_{0}$ and $L_{n}$. This contradicts the minimal choice of $n$.

Thus we have $n=2$ and so $(x, y)$ is a polar pair whose convex closure is a symplecton of polar rank at least 3 . Since $(x, y)$ was an arbitrarily chosen distance 2 point pair, $\Sigma$ is a strong parapolar space of symplectic rank at least 3 . The proof is complete.

Theorem 2.6. Suppose $\Sigma$ is a locally connected parapolar space of symplectic rank at least 3 (but otherwise bounded). Assume that every point-residual $\Sigma(p):=$ $\operatorname{Res}_{\Sigma}(p)$ has the following properties:

(i) All symplecta possess a uniform polar rank $r$. 
(ii) Whenever $S$ is a symplecton and $x$ is a point of $\Sigma(p)$ not in $S$, then $x^{\perp} \cap S$ is either empty or a maximal singular subspace of $S$.

Then $\Sigma$ is a strong parapolar space with uniform symplectic rank $r+1$.

Proof. Assume each point-residual $\Sigma(p)$ of $\Sigma$ satisfies conditions (i) and (ii). Property (i) implies that all symplecta of $\Sigma$ are either quads or have polar rank $r+1$. Since property (ii) holds for each point-residual, we see that for every non-incident point-symplecton pair $(x, S)$ of $\Sigma$, where $S$ is not a quad, $x^{\perp} \cap S$ is equal to the empty set, a single point or a maximal singular space of $S$ which has polar rank at least three. Thus $x^{\perp} \cap S$ cannot be a line. Since $\Sigma$ is locally connected, by Lemma $2.5, \Sigma$ is a strong parapolar space of symplectic rank at least 3. Thus no quads exist. Now condition (i) on point-residuals forces all point-residuals of $\Sigma$ to have the same polar rank $r+1$. The proof is complete.

\subsection{Parapolar spaces, diagram geometries and the local ap- proach theorem}

Consider a geometry with objects whose types belong to a finite set of types $I$, with incidence occurring only between objects of differing types. Equivalently one might view this as a multipartite graph whose component parts are labelled by the set $I$. Here the vertices are the objects of the geometry, sorted by type, and the edges denote the incidences. The number $|I|$ of distinct types of objects, is called the rank of the geometry. A flag of type $J$ is a clique in this graph whose vertex types (one for each vertex) exhaust the types in $J$. The residue of a flag $F$ of type $J$ is the collection of objects of types $I \backslash J$ which are incident with every object in $F$. The flag $F$ is said to be of cotype $I \backslash J$. The geometry is said to belong to a diagram $D$ if the residue of every flag of cotype $\{i, j\}$ (i.e., a residue of type $\{i, j\})$ is specified.

In this paper we shall only be concerned with simply-laced diagrams in which all residues of type $\{i, j\}$ are either projective planes or digons (where every object of one type is incident with every object of the other type). The associated diagram is built this way: its nodes represent the types. Two nodes of types $i$ and type $j$ are connected if and only if the residues of types $\{i, j\}$ are projective planes.

This is a convenient way to represent the structure of a geometry. For example, the proper subspaces of a projective space over a division ring form a diagram geometry $A_{n}$ and similarly, the non-empty singular subspaces of the oriflamme polar spaces form a geometry belonging to the diagram $D_{n}$ (using the names of the familiar Dynkin diagrams). 
Without rehashing well-known definitions, we may distinguish the building geometries which are residually connected diagram geometries of "type $M$ " (meaning that all rank two residues are generalized polygons whose pointdiameter is determined by the type of the residue) and possessing a system of apartments subject to certain axioms. Their importance here lies in the fact that they are universal objects in categories of diagram geometries of type $M$. This is essentially the content of the famous Tits Local Approach Theorem [12].

Remark 2.7. Buildings are normally defined in the category of residually connected chamber systems of type $M$ where 2-covers are easily defined. There Tits's theorem asserts that a building of type $M$ is the universal 2-cover of any chamber system whose rank 3 residues are covered by buildings. But the categories of residually connected chamber systems and residually connected geometries are connected by a functorial equivalence allowing a special case of Tits's theorem to be restated in the context of appropriate diagram geometries.

We state a special case of that theorem.

Theorem 2.8. Suppose $G$ is a diagram geometry with a simply-laced diagram $D$. Then $G$ is a homomorphic image of a building geometry $B$ with the same diagram $D$. More precisely, $G \simeq B / A$ where $A$ is a group of type-preserving automorphisms of $B$ which act freely on chamber-flags of $B$.

Now suppose we have a diagram geometry $G$ with diagram $D$. From such a geometry, one may produce a point-line geometry $\Gamma=(\mathcal{P}, \mathcal{L})$ by selecting as the set of points all objects of a fixed type $t \in I$. The lines are the flags whose type set is that of the set of neighbors of node $t$ in the diagram. In this case we say that the point-line geometry $G$ is derived from or is supported by the diagram $D$. Of course this can be done in a number of ways depending on the choice of $t$ in the diagram.

Remark 2.9. Geometries $G$ with diagram $D_{n}, E_{6}, E_{7}, E_{8}$ are already building geometries. The reason is that these geometries support a parapolar space $\Sigma$ which is either a polar space or a long-root geometry, each of which is already a truncation of a building geometry. (This follows from the fact that the pointcollinearity graph $\Delta$ of a long-root geometry is $\mathcal{T}$-simply connected, where $\mathcal{T}$ is the class of all triangles of $\Delta$; see [6] or [9, Theorem 17.2.8].)

Although a parapolar space $\Sigma$ is defined as a geometry of points and lines, we may also regard it as a geometry $\Sigma^{*}$ further enriched by symplecta and singular subspaces of various projective dimensions to obtain a geometry of higher rank. It may then appear from examining the residuals of each of the objects of $\Sigma^{*}$ that one is seeing a truncation of a diagram geometry with diagram $D$. In this case one must ask if $\Sigma^{*}$ is really a truncation of a diagram geometry? 
The answer is affirmative if the following two conditions are satisfied:

(1) Objects of apparently distinct type within a point-residual $\Sigma^{*}(p)$ are never fused in $\Sigma^{*}$, i.e., they represent the same type of object in $\Sigma^{*}{ }^{4}$ (The factor geometry of Theorem 1.1 is a perfect example of fusion.)

(2) A sheaf exists for the geometry $\Sigma^{*}$. (See [9, Chapter 11].) $)^{5}$

Condition (1) holds if the diagram $D$ has no non-trivial symmetries.

Condition (2) must hold in the particular case that the diagram $D$ has a node of valency three, connecting three separate branches of the diagram, such that it and its three neighbors are types of objects in $\Sigma^{*}$. (See [9, Theorem 11.3.2] for the case that one branch has length one, but the theorem works if that branch is replaced by a string of length more than 1.) It then follows that $\Sigma^{*}$ is a truncation of a homomorphic image of a building geometry with diagram $D$. This theory, which has its antecedents in papers of M. Ronan [7] and A. Brouwer and A. Cohen [1], is fully expounded in [9, Chapter 11].

\section{The geometries of the conclusion of Theorem 1.3}

\subsection{The geometries of Theorem 1.3 for $k=3$}

These geometries, $A_{n, k}$ and $A_{2 n-1, n} /\langle\sigma\rangle$, are strong parapolar spaces whose symplecta are of type $A_{3,2}$. If $(x, S)$ is any non-incident point-symplecton pair, then $x^{\perp} \cap S$ is either empty, a point, or a maximal singular subspace of $S$. So the axiom (H) holds.

An important detail is the following:

Lemma 3.1. For any non-incident point-symplecton pair $(x, S)$ of the line Grassmannian, $A_{n, 2}, x^{\perp} \cap S$ cannot be a single point.

The factor geometries $A_{2 n-1, n} /\langle\sigma\rangle$, though locally well-behaved, have not been fully studied. We can say this much about the point-collinearity graph:

Lemma 3.2. Suppose $\Sigma=A_{2 n-1, n} /\langle\sigma\rangle$ where $\sigma$ is a polarity of $A_{2 n-1, n}$ of Witt index at most $n-5$. Then the geometry $\Sigma$ possesses two points at distance five or more.

\footnotetext{
${ }^{4}$ This notion can be made precise. Two objects $A$ and $B$ of distinct apparent types in $\Sigma^{*}(p)$ are fused in $\Sigma^{*}$ if there is a circuit $\left(p=x_{0}, x_{1}, \ldots, x_{n}=p\right)$ in the point-collinearity graph $\Delta$, and a sequence of objects $\left(A=X_{1}, \ldots, X_{n}=B\right)$ such that $X_{i+1}$ and $X_{i}$ are objects of the same apparent type in the point-residual $\Sigma^{*}\left(x_{i}\right), i=1, \ldots, n$.

${ }^{5}$ There is also the mild condition that $\Sigma^{*}$ be a residually connected geometry, i.e., all its residues of rank 2 or more are connected.
} 
Proof. Let $V$ be the $2 n$-dimensional vector space and let $P$ denote the full collection of $n$-dimensional subspaces of $V$, so that $P$ is essentially the set of "points" of the Grassmann space $\Sigma=A_{2 n-1, n}$. The polarity $\sigma$ can be regarded as a permutation of the subspaces of $V$, taking each $k$-dimensional subspace to a $(2 n-k)$-dimensional space. Notice that $P=P^{\sigma}$. The condition on the Witt index of $\sigma$ now simply means that for every subspace $A \in P, A \cap A^{\sigma}$ has codimension at least 5 in $A$ (and $A^{\sigma}$ ). Among all choices of $A \in P$, we choose $A$ so that the codimension $d$ of $B=A \cap A^{\sigma}$ in $A$ is as small as possible. Notice that $d \geq 5$ and that $B^{\sigma}=\left\langle A, A^{\sigma}\right\rangle$. Thus $W=B^{\sigma} / B$ is a $2 d$-dimensional vector space admitting the action of $\sigma$ on its subspaces so as to induce a polarity $\sigma_{W}$ of the associated projective space $\mathrm{PG}(W)$. Note that this polarity $\sigma_{W}$ has Witt index zero. Again we regard $\sigma_{W}$ as a permutation of the subspaces of $W$. We let $P_{W}$ denote the elements of $P$ which contain $B$ and lie in $B^{\sigma}$. If $X \in P_{W}$, we write $\bar{X}$ for the subspace $X / B$ of dimension $d$. Now, $\bar{A} \cap \bar{A}^{\sigma}=\overline{0}$, the trivial subspace of $W$. It is then easy to construct a third subspace $\bar{C}$ of $W$ having dimension $d$ and intersecting both $\bar{A}$ and $\bar{A}^{\sigma}$ at $\overline{0}$. Then $\bar{C}^{\sigma}$ also intersects $\bar{A}$ and $\bar{A}^{\sigma}$ at $\overline{0}$. Thus, as elements of $\Sigma$, the four Grassmann points $\left\{A, A^{\sigma}, C, C^{\sigma}\right\}$, are all at mutual distance $d$. It follows that $\left(A, A^{\sigma}\right)$ and $\left(C, C^{\sigma}\right)$ are two points of the geometry $\Sigma=A_{2 n-1, n} /\langle\sigma\rangle$ at distance $d \geq 5$. The proof is complete.

\subsection{The $Y_{1}$-geometries and their derivative geometries}

These geometries are the parapolar spaces whose point-residuals are one of the parapolar spaces in the conclusion of Theorem 1.1. Thus, for a given point $p$, any projective plane on $p$ lies in, and is the intersection of, exactly two maximal singular spaces of projective dimensions $a+2$ and $b+2$. If $x$ is a point collinear with $p$, then the line $x p$ lies in some plane that is in exactly two maximal singular spaces of dimension $a+2$ and $b+2$, and so this is true for all planes on $x$. Since the point collinearity graph of a parapolar space is connected, all planes lie in just two maximal singular spaces of these dimensions.

Thus our parapolar space $\Sigma$ enjoys the following local properties.

(L1) Every projective plane lies in exactly two maximal singular subspaces, one a PG $(a+2)$, the other a $\mathrm{PG}(b+2)$, where we can assume $a \leq b$. Moreover, that plane is the intersection of those singular spaces.

(L2) All symplecta are of type $D_{4,1}$. If $p$ is a point not in a symplecton $S$, then $p^{\perp} \cap S$ is either empty, contains a single point, or is a maximal singular subspace of $S$. (Thus the axiom (H) holds.)

(L3) If $p$ is a point not in a maximal singular subspace $M$, then $p^{\perp} \cap M$ is either empty, is a single point, or is a projective plane. 
We divide these geometries into two classes:

Class I: All point-residuals are isomorphic to the Grassmann space $A_{a+b+1, a+1}$, $a \leq b$.

Class II: $a=b$ and there exists a point $p$ whose residual $\operatorname{Res}_{\Sigma}(p)$ is the geometry $A_{a+b+1, a+1} /\langle\sigma\rangle$, where $\sigma$ is a polarity of the underlying PG(2a+1) of Witt index at most $(a+b+1)-5$.

Suppose $\Sigma$ is a parapolar space of Class I. Now if $a=b=1$ then every pointresidual of $\Sigma$ is a polar space and so lies in a symplecton of $\Sigma$. This forces each point-residual to lie in the same symplecton and so $\Sigma$ would be a polar space in that case. But that contradicts the assumption that $\Sigma$ is a parapolar space. Also if $1<a \leq b$, then the common Grassmann space of a point-residual of $\Sigma$ has point diameter at least $a+1$. Thus we have:

Lemma 3.3. If $\Sigma$ is a geometry of Class I, with common residuals of type $A_{a+b+1, a+1}$, then:

(1) $a+b>2$.

(2) $\Sigma$ is a strong parapolar space if and only if $a=1$.

(3) If $a>2$, $\Sigma$ has special pairs.

(4) If $a>3, \Sigma$ has extreme pairs.

Next we distinguish two further classes among the parapolar spaces of Class I:

Class I.1: All pairs of objects of distinct type in the geometry of a point-residual represent objects of distinct types in the geometry $\Sigma$.

Class I.2: Some objects of apparently distinct types in a point-residual, are objects of the same type in $\Sigma$.

There is then a way to tell if a parapolar space $\Gamma$ of Class I with $a=b$ belongs to Class I.1 or to Class I.2. Let $\mathcal{M}$ be the class of all maximal singular subspaces of $\Gamma$. We say that two distinct members of $\mathcal{M}$, say $M_{1}$ and $M_{2}$, are adjacent if and only if $M_{1} \cap M_{2}$ is a line, and we write $M_{1} * M_{2}$ in that case. Now form the graph $(\mathcal{M}, *)$. Note that for every point $p$ in $\Gamma$, the subgraph induced on $\mathcal{M}_{p}$, the collection of maximal singular subspaces incident with $p$, has exactly two connected components: those of the Grassmann residual. Since $\Gamma$ is connected, the graph $(\mathcal{M}, *)$ has at most two connected components.

(D) $\Gamma$ is in Class I.1 or Class 1.2 according as the graph $(\mathcal{M}, *)$ has two or one connected components, respectively. 


\subsubsection{The geometries of Class I.1}

Now consider the enriched geometry $\Sigma^{*}$, consisting of the points, lines, and two classes of maximal singular subspaces of $\Sigma$, whose relationships are described by a locally truncated diagram $Y_{1, a, b}$, truncated to the central node and its three neighbors. Since the two classes of maximal singular subspaces are projective spaces, the appropriate rigidity conditions are in place, allowing us to produce a sheaf and to conclude that $\Sigma^{*}$ is indeed the truncation of a diagram geometry $G$ with diagram $Y_{1, a, b}$. As noted in the paragraph preceding Lemma 3.3, we cannot have $a=b=1$ for in that case $\Sigma$ is a polar space rather than a parapolar space. Since $Y_{1, a, b}$ is a "simply laced" diagram, $G$ is the homomorphic image of a building geometry $B$ by Tits's local approach theorem [12]. Put precisely, $\Sigma$ is a truncation to points and lines, of a geometry $B / A$ where $A$ is a group of automorphisms of the building geometry $B$ acting freely on its flag chambers. Now from our discussion in Subsection 2, $A$ is the identity group if the $Y_{1, a, b}$-diagram is one of the following Dynkin diagrams: $D_{n}, E_{6}, E_{7}$ or $E_{8}$. In those cases, the point-line geometry $\Sigma$ is respectively isomorphic to (1) a half-spin geometry, (2) the long-root geometry $E_{6,2}$, (3) the geometry $E_{7,2}$, and (4) $E_{8,2}$. The last two have never before made an appearance in a characterization theorem in parapolar spaces.

Note that the common Grassmann space of our point-residuals has point diameter $a+1$. As a result, if $a>1$, the parapolar space $\Sigma$ contains special pairs, and contains extreme pairs if $a>2$.

\subsubsection{The geometries of Classes I.2 and II}

Suppose $\Sigma$ is a parapolar space of Class I.1. If two objects $A$ and $B$ of distinct types in a point-residual represent the same object of the enriched geometry $\Sigma$, the two objects must be isomorphic subspaces of different types. This is only possible if the point-residual $\operatorname{Res}_{\Sigma}(p)$ is isomorphic to the Grassmannian of type $A_{2 a+1, a}$, that is to say $b=a$. If $a=2$, special pairs are present, and if $a>2$ extreme pairs are present.

The geometries of Class I.1 exist (they are point-line geometries derived from a homomorphic image of a building geometry) but it is not clear that parapolar spaces of Class I.2 even exist. Using Lemma 3.2, we see that all geometries in Class II, if any exist, are extreme parapolar spaces. 


\section{Proof of the Main Theorem}

\subsection{Introductory remarks}

The proof of Theorem 1.2 given in [4] is a "bottom-up" proof. That is, because of the constant symplectic rank, the proof could begin with the case that the uniform symplectic rank $r$ of the parapolar space was three (basically Theorem 1.1), then consider the cases $r=4,5, \ldots$, one after another, each time armed with full knowledge of the point-residuals. The procedure finally terminates with $r=6$, when one reaches $E_{7,7}$ which has point-diameter three and so cannot be the point-residual of a further strong parapolar space.

Such an approach is not possible here because one is immediately confronted with a population of symplecta with a multitude of polar ranks. Instead we take a "top-down" approach beginning with the class $\mathcal{A}_{2}$ of singular subspaces which have codimenion 2 in a maximal singular subspace of any symplecton of maximal polar rank $n$ in the parapolar space. For $A_{2} \in \mathcal{A}_{2}$, the residue $\Sigma_{2}:=\operatorname{Res}_{\Sigma}\left(A_{2}\right)$ is a point-line geometry of some sort. We do not know if it is even connected. All we know at the outset is that it possesses a non-empty class of symplecta, all of uniform polar rank two with the haircut axiom $(H)$, but even then, it is not obvious that every line lies in such a rank 2 symplecton. However, using $(\mathrm{H})$ we will show that all these objections will fall away.

Let $\Sigma$ be a parapolar space satisfying the hypotheses of the Main Theorem. By hypothesis, there is an upper bound on the possible ranks of a symplecton of $\Sigma$. We let $\mathcal{S}$ denote the class of symplecta which reach this maximal polar rank $n$. In general, we let $\mathcal{A}_{k}$ denote the set of all singular subspaces which have codimension $k$ in a maximal singular subspace of a symplecton of $\mathcal{S}$. For each $A_{k} \in \mathcal{A}_{k}$, we obtain a point-line geometry $\operatorname{Res}_{\Sigma}\left(A_{k}\right)$ whose points and lines are all the singular subspaces of $\Sigma$ in which $A_{k}$ has codimension 1 or 2 , respectively. Notice that if $A_{k}=\emptyset$, the empty subspace, then $\operatorname{Res}_{\Sigma}\left(A_{k}\right)=\Sigma$.

Lemma 4.1. For $A_{k} \in \mathcal{A}_{k}, k \geq 2$, the point-line geometry $\Sigma_{k}=\operatorname{Res}_{\Sigma}\left(A_{k}\right)$ is connected.

Proof. Let $\Gamma_{k}$ be a connected component of $\Sigma_{k}$. Clearly $\Gamma_{k}$ is a subspace of $\Sigma_{k}$. We assume by way of contradiction that $\Sigma_{k}$ is not connected.

If $A_{k}=\emptyset$, that is, $\Sigma_{k}=\Sigma$, then it is a parapolar space and so by definition is connected. Thus we may assume $A_{k} \neq \emptyset$. Thus there is a (possibly empty) singular space $A_{k+1}$ of codimension 1 in $A_{k}$. If $A_{k+1}=\emptyset$, so that $\Sigma_{k+1}=\operatorname{Res}_{\Sigma}\left(A_{k+1}\right)=\Sigma$, then by hypothesis, $\Sigma_{k+1}=\Sigma$ is locally connected, forcing $\Sigma_{k}$ to be connected against our assumption. Thus we may also assume 
$A_{k+1} \neq \emptyset$. Thus we also have (again a possibly empty) singular subspace $A_{k+2}$, of codimention 1 in $A_{k+1}$.

Since $\Sigma_{k}$ is not connected, there exists a "point" $B$ of $\Sigma_{k}$ not in $\Gamma_{k}$ and a "point" $C$ in $\Gamma_{k}$. Now consider these spaces as objects of the geometry $\Sigma_{k+2}$. In this geometry $A_{k+1}$ is a "point," $A_{k}$ is a "line," and $B$ and $C$ are two "planes" which intersect at "line" $A_{k}$ and together do not lie in a singular subspace (otherwise $B$ and $C$ would be collinear in $\Sigma_{k}$, contrary to our choice of $C$ and $B$ ). It follows from the parapolar space axioms that $B$ and $C$ are in a symplecton of $\Sigma_{k+2}$ of polar rank three, and so, in $\Sigma_{k+1}$ represent two "lines" of a convex generalized quadrangle which we denote $Q$.

The rest of the argument proceeds within the geometry $\operatorname{Res}_{\Sigma}\left(A_{k+1}\right)$. From the foregoing we see that the "lines" $B$ and $C$ of $\Sigma_{k+1}=\operatorname{Res}_{\Sigma}\left(A_{k+1}\right)$ meet at "point" $A_{k}$ and lie in a quad $Q$ which meets $\Gamma_{k}$ at "line" $C$. There exists a "line" $E$ containing "point" $A_{k}$ (representing a "point" of $\Gamma_{k}$ ) which does not lie in a singular subspace with $C$. (This is because $\Gamma_{k}$ contains a non-degenerate symplecton.) Choose a "point" $X$ in "line" $E$ distinct from "point" $A_{k}$. Then (in $\left.\Sigma_{k+1}\right), X^{\perp} \cap Q$ contains the "point" $A_{k}$. But $Q$ possesses the haircut property (H) forcing $X^{\perp} \cap Q=M$, a line of the quad $Q$. The "line" $M$ is distinct from $C$ since the latter is not in $X^{\perp}$.

But now (shifting from $\Sigma_{k+1}$ to $\Sigma_{k}$ ), $M$ becomes a "point" of $\Sigma_{k}$ not in $\Gamma_{k}$ yet "collinear" to the "point" $E$ of $\Gamma$. That contradicts the fact that $\Gamma_{k}$ is a connected component of $\Sigma_{k}$. The proof is complete.

Corollary 4.2. For any $A_{k} \in \mathcal{A}_{k}, k \geq 3$, the geometry $\Sigma_{k}=\operatorname{Res}_{\Sigma}\left(A_{k}\right)$ is not itself a symplecton.

Proof. We know that when $m$ is the maximal polar rank of a symplecton, then $A_{m}=\emptyset$, so that $\Sigma_{m}=\Sigma$ is a parapolar space and so by definition is not itself a polar space. Suppose, for some $k, \Sigma_{k}$ is a polar space. Then this is true for $\Sigma_{j}$ for all $j$ with $3 \leq j \leq k$. We may then assume $k$ is chosen as large as possible such that $\Sigma_{k}$ is a polar space. Now, as observed at the beginning of this paragraph, $A_{k}$ contains a hyperplane $A_{k+1}$. Moreover, the singular spaces in $\Sigma_{k+1}$ which contain its "point" $A_{k}$ must lie in a symplecton $R$ of polar rank $k+1$. But from our choice of $k, \Sigma_{k+1} \neq R$. But, from Lemma 4.1, $\Sigma_{k+1}$ is connected and so it contains a "point" $B$ not in $R$ collinear with a "point" of $R$, which, without loss of generality, we may take to be $A_{k}$. Now the singular spaces of $R$ which contain $A_{k}$ form the unique symplecton of $\Sigma_{k}$. But that is impossible, since the space $\left\langle B, A_{k}\right\rangle$ represents a "point " of $\Sigma_{k}$ not in that symplecton. The proof is complete. 
Lemma 4.3. If $A_{k} \in \mathcal{A}_{k}$, and $A_{k} \neq \emptyset, k \geq 2$, then every geodesic path of length two in $\Delta_{k}$, the point-collinearity graph of $\Sigma_{k}=\operatorname{Res}_{\Sigma}\left(A_{k}\right)$, lies in a symplecton of $\Sigma_{k}$. Moreover, each line of $\Sigma_{k}$ lies in a symplecton.

Proof. Let $(A, B, C)$ be a shortest path connecting two points $A$ and $C$ in $\Sigma_{k}$. Then, as $A_{k} \neq \emptyset, A_{k}$ contains a (possibly empty) hyperplane $A_{k+1} \in \mathcal{A}_{k+1}$. Then in the point-line geometry $\Sigma_{k+1}=\operatorname{Res}_{\Sigma}\left(A_{k+1}\right), A, B$, and $C$ are three lines on the point $A_{k}$, the first two and last two of these lines spanning planes while $A$ and $C$ do not lie in a plane. Then, from the parapolar space axioms for $\Sigma, A$ and $C$ are lines of $\Sigma_{k+1}$ lying in a symplecton $R$ of $\Sigma_{k+1}$ of polar rank at least three. Now the lines and planes of $R$ which contain $A_{k}$ form a symplecton of $\Sigma_{k}$ containing the path $(A, B, C)$.

It remains to show that any line of $\Sigma_{k}$ lies in a symplecton. Now suppose $A, B$ are two collinear points of $\Sigma_{k}$. By the result of the previous paragraph, if $(A, B)$ belongs to a geodesic path $(D, A, B)$ or $(A, B, C)$, we are done.

Since $\Sigma_{k}$ contains a symplecton, we cannot have $\Sigma_{k}=A^{\perp}$. Thus there is a point $C$ not collinear with $A$, and since $\Sigma_{k}$ is connected by Lemma 4.1, we can assume $C$ is collinear with a point $E$ in $A^{\perp}$. Then $(A, E, C)$ is a geodesic path of length two and so, by the previous paragraph, this path lies in a symplecton $R$ of polar rank at least 2. If $R$ contains $B$ we are done. Thus we may assume that $B$ is not in $R$. Now $B^{\perp} \cap R$ is a clique containing $A$, while $A^{\perp} \cap R$ is not a clique. Thus in $R$ there is a point $B \in A^{\perp}$ which is not collinear with $B$. Thus $(D, A, B)$ is now a geodesic path of length two and so it must lie in some symplecton. Thus the line on $(A, B)$ does lie in a symplecton. The proof is complete.

Corollary 4.4. For $A_{k} \in \mathcal{A}_{k}, k \geq 3$, the point-line geometry $\Sigma_{k}$ is a parapolar space. If $A_{k} \neq \emptyset$ it is a strong parapolar space.

Proof. The statement is true by hypothesis if $A_{k}=\emptyset$ so that $\Sigma_{k}=\Sigma$. So we may assume that $A_{k} \neq \emptyset$. Then the result follows from Lemma 4.1, Lemma 4.3 and Corollary 4.2.

With these preliminaries in place, we may proceed with the proof of the main theorem in the next series of subsections. From this point onward assume that $\Sigma$ satisfies the hypotheses of Theorem 1.3.

\subsection{The case $k=3$}

Lemma 4.5. If $A_{3} \in \mathcal{A}_{3}$, then $\Sigma_{3}$ is either a Grassmann space $A_{n, k}$ or the factor space $A_{2 n-1, n} /\langle\sigma\rangle$ where $\sigma$ is a polarity of $\mathrm{PG}(2 n-1)$ of Witt index at most $n-5$. 
Proof. Before we can apply Cohen's theorem there are some things to prove. First, consider a space $A_{2} \in \mathcal{A}_{2}$ so that $A_{3} \subseteq A_{2}$ and set $\Sigma_{2}=\operatorname{Res}_{\Sigma_{3}}\left(A_{2}\right)$. Now by Corollary 4.4 both $\Sigma_{3}$ and $\Sigma_{2}$ are parapolar spaces and so are connected. Since all point residues of $\Sigma_{3}$ are connected, $\Sigma_{3}$ is locally connected.

Now by (possibly repeated) application of Lemma 2.4, both $\Sigma_{3}$ and $\Sigma_{2}$ possess the haircut property $(\mathrm{H})$. Thus for any symplecton of $\Sigma_{2}$, some quad $Q$, and point $x$ not in $Q$, we have that $x^{\perp} \cap Q$ is either empty or a maximal singular subspace of $Q$ (i.e., a line). Since $\Sigma_{2}$ is any typical point-residual of $\Sigma_{3}$, we have gathered together the hypotheses of Theorem 2.6 with the parameter $k=2$. Thus we conclude that $\Sigma_{3}$ is a strong parapolar space of uniform symplectic rank three. Now we may apply Theorem 1.1 to reach the conclusion. The proof is complete.

\subsection{The case $k=4$}

Choose $A_{4} \in \mathcal{A}_{4}$ and let $\Sigma_{4}=\operatorname{Res}_{\Sigma}\left(A_{4}\right)$. For each point $p$ in the parapolar space $\Sigma_{4}$, the point-residual $\operatorname{Res}_{\Sigma_{4}}(p):=\Sigma_{4}(p)$ is the Grassmannian $A_{n, k}$, or the factor geometry $A_{2 a+1, a+1} /\langle\sigma\rangle$ as described in Theorem 1.1. Now this means that every plane of $\Sigma_{4}$ that contains the point $p$ lies in exactly two maximal singular subspaces of projective dimensions $k$ and $n-k+1$ (or two singular spaces of the same dimension $k=a+1$ in the factor geometry case). Now if $q$ is another point of $\Sigma_{4}$ collinear with $p$, the line $p q$ lies in a plane whose two maximal singular subspaces above it have the same dimensions "as for $p$." Since $\Sigma_{4}$ is a connected geometry, it now follows that every plane of $\Sigma_{4}$ lies in exactly two maximal singular subspaces of projective dimensions $n$ or $n-k+1$. When these two numbers are distinct every point-residual $\Sigma_{4}(p)$ is isomorphic to $A_{n, k}$. But when the two numbers are equal it is possible that $\Sigma_{4}(p)$ is $A_{2 a+2, a+1}$ for some choices of point $p$ and is $A_{2 a+1, a+1} /\langle\sigma\rangle$ for other choices.

We must next show that $\Sigma_{4}$ has uniform symplectic rank. Since all the pointresiduals $\Sigma_{4}(p)$ have constant symplectic rank three, all of the symplecta of $\Sigma_{4}$ are either of polar rank 4 or else they are quads. We need only show that no quads can be present. There are two cases.

The case $A_{4}=\emptyset$, that is $\Sigma=\Sigma_{4}$ : in this case $\Sigma=\Sigma_{4}$ has symplectic rank at least three by hypothesis, and so no quads are present. By definition, $\Sigma_{4}$ is one of the $Y_{1}$-geometries or one of their twisted versions in Class I.2 or Class II, loosely described in Subsection 3.2 above.

The case $A_{4} \neq \emptyset$ : in this case, $\Sigma_{4}$ is itself a residue of a non-empty singular space $A_{4}$, and so is a strong parapolar space. But in that case, by Lemma 2.3(3), each of its point-residuals are parapolar spaces of point-diameter 2 . We already know that if $3 \leq k \leq n / 2$, this Grassmann space has point-diameter at least $k$. In 
the case of the $A_{2 a+1, a+1} /\langle\sigma\rangle$, the point-diameter is at least five (Lemma 3.2). Thus we see that all point-residuals in $\Sigma_{4}$ are isomorphic to the same lineGrassmannian $A_{n, 2}$. But in this case, if $(\bar{x}, \bar{S})$ is a non-incident point-symplecton pair of $\Sigma_{4}(p)$, then $\bar{x}^{\perp} \cap \bar{S}$ is either empty or a maximal singular subspace of $\bar{S}$, a plane. Noting that each $\Sigma_{4}(p)$ is connected, we see that $\Sigma_{4}$ is locally connected. We now have all the hypotheses of Theorem 2.6 in place, with $\Sigma_{4}$ in the role of the $\Sigma$ of that theorem and the parameter $k$ of that theorem equal to three. We therefore conclude that $\Sigma_{4}$ is a strong parapolar space of uniform symplectic rank 4.

Now the rank 4 geometry of points, lines and the two distinct classes of maximal singular spaces (an enrichment of $\Sigma_{4}$ ) possesses a chamber connected sheaf by Theorem 11.3.2 of [9], and so is the truncation of a homomorphic image of a building geometry with the diagram $D_{n}$ (see [9, Theorem 11.7.1]). Since a building geometry of type $D_{n}$ supports a polar space this homomorphism is an isomorphism. In this case $\Sigma_{4}$ is a half-spin geometry of type $D_{n, n}, n \geq 5$.

\subsection{The case $k=5$}

Here we choose any $A_{5} \in \mathcal{A}_{5}$ and set $\Sigma_{5}=\operatorname{Res}_{\Sigma}\left(A_{5}\right)$. Now for each point $p$, the point-residual $\Sigma_{5}(p)$ is a strong parapolar space of the form $\operatorname{Res}_{\Sigma}\left(A_{4}\right)$ for some $A_{4} \in \mathcal{A}_{4}$. By the previous subsection, $\Sigma_{4}(p)$ is isomorphic to $D_{n, n}$ for some $n$ that may depend on $p$. But the parameter $n$ is determined by the largest dimension, $n-1$, of a singular subspace of $\Sigma_{5}(p)$ and so is shared by the pointresiduals of all its neighbors in the point-collinearity graph of $\Sigma_{5}$. Since the latter is connected, all point-residuals of $\Sigma_{5}$ are half-spin geometries $D_{n, n}$ for fixed $n$.

At this point $\Sigma_{5}$ is a parapolar space whose symplecta are either quads or have polar rank 5 . We will show that $\Sigma_{5}$ has constant symplectic rank 5 by an argument similar to that of the previous subsection.

First, if $A_{5}=\emptyset$ so that $\Sigma_{5}=\Sigma$, then no quads can exist since $\Sigma$ has symplectic rank at least three by hypothesis.

So we consider the case that $A_{5} \neq \emptyset$. In this case $\Sigma_{5}$, being the residue of a nontrivial subspace $A_{5}$, is a strong parapolar space. In this case, its pointresiduals must have point-diameter two. But this is only possible if all pointresiduals are of the same type $D_{5,5}$. These geometries possess the property that for any non-incident point-symplecton pair $(\bar{x}, \bar{S}), \bar{x}^{\perp} \cap \bar{S}$ is either empty, or is a maximal singular subspace of $\bar{S}$. Now, since all point-residuals are connected and have constant symplectic rank, we may apply Theorem 2.6 to conclude that $\Sigma_{5}$ has constant symplectic rank 5 , and that $x^{\perp} \cap S$ is either empty, a single point or a maximal singular subspace. It follows from Theorem 2 of Cohen and 
Cooperstein [4], that $\Sigma_{5}$ is the Lie incidence geometry $E_{6,1}$ in this case.

Let us return to the general case, where $\Sigma=\Sigma_{5}$ and every point-residual is the geometry $D_{n, n}$ for fixed $n \geq 5$. Here, when $n>5, \Sigma$ is not a strong parapolar space, and when $n>7$, it is extreme. Now, by the same sheaf-theoretic arguments encountered in the case $k=4$, we see that $\Sigma_{5}$ is a truncation of a homomorphic image of the building geometry with diagram $E_{n+1}$. When $n=6,7$ or 8 , the building geometries support a long-root geometry and so the homomorphism in question is an isomorphism in these cases yielding $\Sigma$ isomorphic to $E_{6,1}, E_{7,1}$ and $E_{8,1}$. For $n>8$, a proper homomorphism of a building geometry with diagram $E_{n}$ may support $\Sigma$. In these cases, the resulting parapolar space is extreme. Only in the case of $E_{6,1}$ is the geometry a strong parapolar space.

\subsection{The case $k=6$}

Again choose $A_{6} \in \mathcal{A}_{6}$ and set $\Sigma_{6}=\operatorname{Res}_{\Sigma}\left(A_{6}\right)$. Now consider any point-residual $\Sigma_{6}(p):=\operatorname{Res}_{\Sigma_{6}}\left(A_{5}\right)$, where $p$ is represented by a space $A_{5} \in \mathcal{A}_{5}$ containing $A_{6}$. Then $\Sigma_{6}(p)$, being a strong parapolar space, must be isomorphic to $E_{6,1}$, by the analysis for the case $k=5$.

Again, we must show that $\Sigma_{6}$ has uniform symplectic rank. From the uniform nature of its point-residuals, we see that the symplecta of $\Sigma_{6}$ are either of type $D_{6,1}$ or are quads.

Now $\Sigma_{6}$ is a locally connected parapolar space whose point-residuals, being isomorphic to $E_{6,1}$, have property (K), so that for any non-incident pointsymplecton pair $(\bar{x}, \bar{S}), \bar{x}^{\perp} \cap \bar{S}$ is either empty, or is a maximal singular subspace of $\bar{S}$. Now Theorem 2.6 implies that $\Sigma_{6}$ is a strong parapolar space of symplectic rank at least three. Thus no quads can be present, and so $\Sigma_{6}$ has uniform symplectic rank.

Since all of the following seven types of objects — points, lines, planes, $\mathrm{PG}(3)$ 's, $\mathrm{PG}(5)$ 's, symplecta, and $\mathrm{PG}(6)$ 's - are present in the enriched geometry $\Sigma_{6}^{*}$ of the parapolar space $\Sigma_{6}$, a perusal of its rank two residues reveals the diagram $E_{7}$. By Remark 2.9, we see that $\Sigma_{6}$ is the strong parapolar space $E_{7,7}$ of point-diameter 3 .

\subsection{The case $k=7$}

For any $A_{7} \in \mathcal{A}_{7}, \Sigma_{7}=\operatorname{Res}_{\Sigma}\left(A_{7}\right)$ is a parapolar space whose enriched pointresiduals are geometries of type $E_{7,7}$. Since these point-residuals have pointdiameter $3, \Sigma_{7}$ cannot be a strong parapolar space (Lemma 2.3(2)). Thus $\mathcal{A}_{8}$ contains only one singular subspace, namely the empty set, forcing $\Sigma=\Sigma_{7}$. 
Since $\Sigma$ has symplectic rank at least three, there are no quads and so all symplecta of $E_{7}$ are of type $D_{7,1}$.

In the enriched geometry $\Sigma_{7}^{*}$ there are 8 apparent types of objects: points, lines, planes, $\mathrm{PG}(3)$ 's, $\mathrm{PG}(4)$ 's, $\mathrm{PG}(6)$ 's, $\mathrm{PG}(7)$ 's and the symplecta.

The $P G(6)$ 's are a class of maximal singular subspaces which lie in a symplecton. The PG(7)'s are also a class of maximal singular spaces, but they do not lie in a symplecton. A PG(7) is incident with a symplecton if and only if it intersects it at one of its maximal singular subspaces (which obviously cannot belong to the class of maximal $\mathrm{PG}(6)$ 's). A maximal $\mathrm{PG}(7)$ is incident with a maximal PG(6) if and only if they intersect at a PG(4). All of the other incidences are containment relationships of subspaces. One then sees that these types of objects form a diagram geometry of type $E_{8}$. From Remark 2.9, $\Sigma$ is the long-root geometry $E_{8,8}$.

Since $\Sigma=\Sigma_{7}$ was forced on us, and there are no further cases, this concludes the proof of Theorem 1.3.

Remark 4.6. The proof of uniform symplectic rank succeeds because of a certain serendipity:

Among the Lie incidence geometries which are strong parapolar spaces of point-diameter 2 (the line Grassmannians, $D_{5,5}$ and $E_{6,1}$ ) it happens that for each non-incident point-symplecton pair $(x, S), x^{\perp} \cap S$ is either empty or a maximal singular subspace of $S$.

The author is unaware of any theoretical reason for this implication among general parapolar spaces.

\section{References}

[1] A. E. Brouwer and A. M. Cohen, Local recognition of Tits geometries of classical type, Geom. Dedicata 20 (1986), 181-199.

[2] A. Cohen, On a theorem of Cooperstein, European J. Combin. 4 (1983), 107-126.

[3] _ Point-line spaces related to buildings, in Handbook of Incidence Geometry (F. Buekenhout ed.), Elsevier, Amsterdam, 1995, pp. 647-737.

[4] A. Cohen and B. Cooperstein, A characterization of some geometries of Lie type, Geom Dedicata 15 (1983), 73-105. 
[5] B. Cooperstein, Some geometries associated with parabolic representations of groups of Lie type, Canadian J. Math. 28 (1976), 1021-1031.

[6] A. Kasikova and E. Shult, Point-line characterizations of Lie geometries, Adv. Geom. 2 (2002), 147-188.

[7] M. Ronan, Extending locally truncated buildings and chamber systems, Proc. London Math. Soc. 53 (1986), 385-406.

[8] J. Schillewaert and H. Van Maldeghem, Imbrex geometries, J. Combin. Theory Ser. A 127 (2014), 286-302.

[9] E. E. Shult, Points and Lines. Characterizing the classical geometries, Springer Verlag, Berlin-Heidelberg, 2011.

[10] _ On characterizing the long-root geometries, Adv. Geom. 10 (2010), 353-370.

[11] E. E. Shult and K. Thas, A theorem of Cohen on parapolar spaces, Combinatorica 30 (2010), 435-444.

[12] J. Tits, A local approach to buildings, in The Geometric Vein (The Coxeter Festschrift; D. B. Grunbaum and F. A. Sherk ed.), Springer Berlin, 2002, pp. 519-547.

[13] O. Veblen and J. Young, Projective Geometry, Ginn, Boston, 1916.

Ernest E. Shult

Kansas State University, Manhattan, Kansas 66502, USA 